\title{
A manufacturing support system for industrial part process planning
}

\author{
Adilson U. Butzke, Joao C. E. Ferreira \\ Federal University of Santa Catarina, \\ Mechanical Engineering Department, GRUCON, CaixaPostal 476, \\ 88040-900, Florianopolis, SC, Brazil, Tel:(+55)(48) 2319387, \\ Fax: (048) 2341519, E-mail: jcf@grucon.ufsc.br
}

\begin{abstract}
Much research work has been carried out (and still is) on the development of Computer-Aided Process Planning (CAPP) systems as a means of bridging the gap between design and manufacture, aiming at an increase in production, higher production quality and lower costs. However, most of these works have been developed at research centers outside the factory. This commonly leads to the resulting CAPP system not being of efficient use on the shop-floor. Described within this paper is the architecture of a Manufacturing Support System for Process Planning, referred to as MSSPP, which is being developed as a cooperation between the Federal University of Santa Catarina (UFSC) and Schneider Logemann Cia. (SLC), a Brazilian company which produces machines for agricultural harvesting and planting. The system will support the process planner in the preparation of consistent and feasible process plans, taking into account the resources available at the shop-floor. It will also provide the simulation of the tool paths for machining.
\end{abstract}

\section{Keywords}

Process Planning, Design and Manufacture, Features, Databases

\section{INTRODUCTION}

Some years ago the industrial sector used to manufacture their goods and launch them into the market independent of the customer's desires. Since there was almost no competition among producers, the customer had no alternative but to purchase the product. With the technological developments on communications, machine tools, cutting tools and computers, there was an increase on competition, and a greater variety of products was available for the 
customer, who obviously would choose the one which was cheaper, and of good quality and reliability. The companies used to be "manufacturer-oriented", and became "customeroriented" [1].

These companies purchased state-of-the-art equipment, and reorganized themselves in order to cope with this new type of production. However, some bottlenecks are usually present after the modernization, which prevent the company from attaining even higher levels of production, together with lower scrap and higher operator satisfaction. One of these bottlenecks is process planning.

Process planning is an activity which takes place in most factories, and is responsible for decisions such as: machine tool selection, tool selection, workholder selection, machining parameter selection and determination of the operations and their sequence. Process planning is therefore a crucial activity in product manufacture. It is traditionally carried out manually, which means that company production depends on the experience of process planners.

The process planning activity, performed in that way, can lead to a longer time for part manufacture. Some of the problems related to that are as follows:

- The process planner must have many years of experience. If him/her retires, a significant amount of time will be needed for the substitute planner to be as productive as his antecedent.

- The amount of information to be considered by the process planner is very high. Even with a vast experience, a long time is spent by him/her on process plan preparation.

- Process planning is traditionally a human activity, and as a result different planners normally prepare different process plans for the same part. Production organization may be difficult due to this inconsistency. Since on customer-oriented production the variety of parts is relatively high, it is difficult to manage these process plans.

- With the available equipment, it may be difficult to manufacture the part. In this case, the process planner would suggest a design change to the designer, so that the part can be manufactured on the shop-floor. This leads to a longer time for part production. Moreover, the different points of view of these professionals may result in clashes between them.

- It is a common practice to file the part drawing together with the process plan associated with each part. In the case of demand for a certain part, the process plan associated with it is followed through. However, this process plan may not be feasible with the resources available at the time, due to reasons such as machine maintenance, machine replacement, tools not available, etc. In this case, the process plan would have to be altered in order to adapt to the available resources. This process takes time, and thus delays part production.

Many companies, despite presenting a certain amount of production organization and automation, and also being susceptible to market change, have the deficiencies presented above, with regard to the important activity of process planning.

Research on Computer-Aided Process Planning (CAPP) systems has started in the 70's, and since then the efforts made in that direction have been academic [2,3], although some researchers report the use of their systems by industry [4,5]. In spite of the benefits attained by the CAPP systems developed to date, very few companies implement these systems. According to ElMaraguy [6], approximately $85 \%$ of all process plans produced in industry today are created manually, and optimized process plans are rarely produced.

Knowing that process planning is a bottleneck in many companies, it is important that a CAPP system is developed in such a way that the culture and organization of the company are taken into account. As a result, a cooperative work has started between the Federal University of Santa Catarina (UFSC) and Schneider Logemann Cia. (SLC), a Brazilian company which 
produces machines for agriculture harvesting and planting, aiming at the development of a Manufacturing Support System for Process Planning, referred to as MSSPP, whose architecture is described within this paper. This system is intended to assist the process planner in choosing the most appropriate process plan for part manufacture. With this system it is expected that the problems pointed out above are reduced or eliminated.

Although the automation of process planning has been aimed at by many authors (e.g. [7-9]), these systems lack an efficient interface between CAD and CAPP. One of the main objectives of MSSPP is to link CAD and CAPP through "feature" technology, i.e., the part is modelled based on features in the CAD module. Since features carry geometrical and technological attributes which aggregate process planning information, such as tools and fixtures, they enable the efficient process planning for the part's manufacture.

\section{METHODOLOGY FOR THE DEVELOPMENT OF THE MSSPP}

The activity of process planning is usually very complex, and that is the case at SLC. In order for the development and implementation of the MSSPP, the process planning problem has been subdivided into smaller problems. This subdivision consisted of: 1-reduction of the process planning domain; 2-decomposition of the system into modules. An explanation of these two strategies is given below.

\section{Reduction of the Process Planning Domain}

SLC produces machined and sheet-metal parts, and for these types of parts there are related departments. Most of the machining department is organized into manufacturing cells, which present advantages such as: shorter material transportation and handling times, easier part finding, etc. Since each cell manufactures a significant amount of parts, they can be considered as "small factories" (see Figure 1). Rarely a part from one cell is introduced into another cell. So, for the present work, one cell has been chosen for the development and implementation of the CAPP system, which is a reduction in the process planning domain. It is expected that the same approach can be applied successfully to other cells.

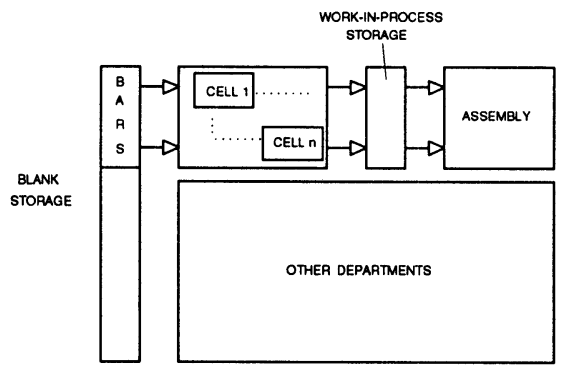

Figure 1 Flow of parts within SLC (Storage $\rightarrow$ Cell $\rightarrow$ Assembly)

It has been chosen a cell where approximately 480 parts are manufactured, and the machine tools in this cell are: four CNC lathes with bar feeder, one conventional Universal lathe, and two sawing machines. The tools used by each machine are placed on shelves beside the machines, as well as the workholders. This reduces transport time of tools and workholders, 
and facilitates their management. The transport of parts, tools and workholders within the cell is done manually.

\section{System Modularization}

In software development, the technique of separating specific tasks into modules usually gives good results, mainly because different groups of people may develop each module, independent of the other modules, which decreases the time for development. Also, if modifications to a specific task are necessary, the related module is altered, without need to change the other modules.

Process planning is an activity where various tasks are performed. According to Wang and Li [1], an effective CAPP system should: 1-communicate with a CAD module, 2-select the blank, 3-select the operations, 4-select machine tools and cutting tools, 5-determine intermediate surfaces, 6-select machining parameters, 7-select workholders, 8-estimate time and cost, and 9-generate the cutter paths

Therefore, it is recommended that a CAPP system should be modular for ease of development and maintenance.

The proposed architecture of this system is shown in Figure 2. This architecture has been defined taking into consideration the attributes pointed out above.

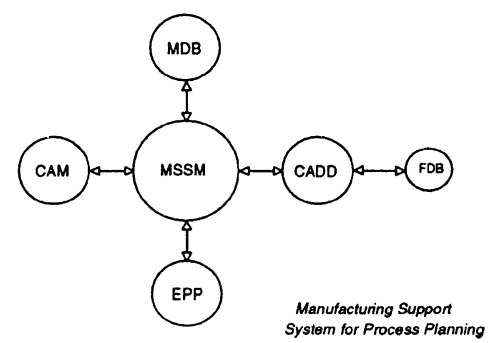
where:
CADD: design and drafting module
FDB: feature database
MDB: manufacturing database
EPP: expert process planning module
CAM: tool path generation and simulation module
MSSM: manufacturing support system manager

Figure 2 Proposed architecture of the Manufacturing Support System for Process Planning

A description of each of the modules, and how they interact with each other is given below.

\section{THE CADD MODULE}

This module has as objective to assist the designer in the construction of the part in the computer. The part representation must be complete and unambiguous, so that the constructed part corresponds to the desired one.

In this module, "features" are used as the building blocks for the parts. Many different feature definitions have been found in the literature, but there has not been a consensus yet. In this work, "features" are defined as "regions or volumes in machined parts, being important for design, process planning and other activities" [10]. Some examples of features in this work are 
shafts, holes, chamfers, grooves, etc. Figure 3 illustrates how process planning information (e.g., machine, tool, workholder) can be selected, having a feature as the origin.

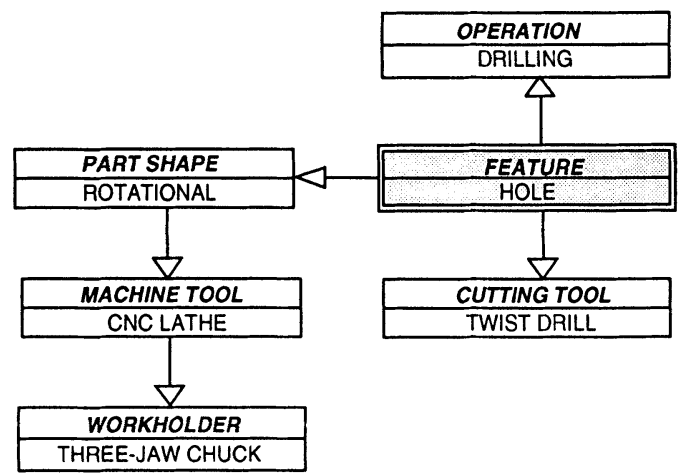

Figure 3 Manufacturing decisions based on features

Two advantages of feature-based design are:

- Features are elements which carry geometrical, functional and technological information, and therefore the designer is more acquainted with them.

- Features facilitate reasoning about the manufacturability of the part, and consequently its process plan.

Much research work has been developed on feature-based design (e.g. [11,12]), and features have been used in many CAPP systems, such as TURBO-MODEL [7], FIRST-CUT [13] and Juri's system [14].

In the TURBO-MODEL system [7], the methodology applied consists of representing various types of information about the part (i.e., geometrical, technological and global information). It models rotational parts, and part's design is carried out in a pre-determined way. That system includes a sub-program to verify the feasibility of the instantiated features, with respect to geometrical, technological and functional attributes. It also has an interface with AutoCAD. A limitation of that system is that it does not provide much flexibility to the designer during feature-instantiation.

FIRST-CUT [13] is a feature-based design system that aims at integrating CAD and CAPP. In this system, the process analysis for product manufacture is incorporated into the design phase, in order to attain an efficient process plan.

Juri [14] investigated the feature-based approach for modelling rotational parts in a product modelling system. A product is modelled through form features, which can be related to a manufacturing process, and a structure based on frames is used to represent the features. A technique based on production rules is applied in order to perform operation selection and sequencing.

Since the design methodology at SLC is based on features, the CADD module is featurebased, and thus a feature database (FDB) has been constructed. In order to construct the feature database, information has been gathered on the features present in the drawings of the parts machined at the cell. Based on this information, features have been classified, and a portion of this classification is illustrated in Figure 4. This classification is based on the geometry of the features, as it has been done by Jasthi [7]. 
Detailed information on two features present in parts at SLC is given in Figure 5. Notice that in order to decrease time for search and disk space usage, the features are parameterized. It should also be noticed that none of the geometric constraints shown in the figure can be violated, otherwise the part would be inconsistent. This is a means of making the software robust and reliable.

In order to construct a part, the designer must go through the steps described in the pseudocode below:
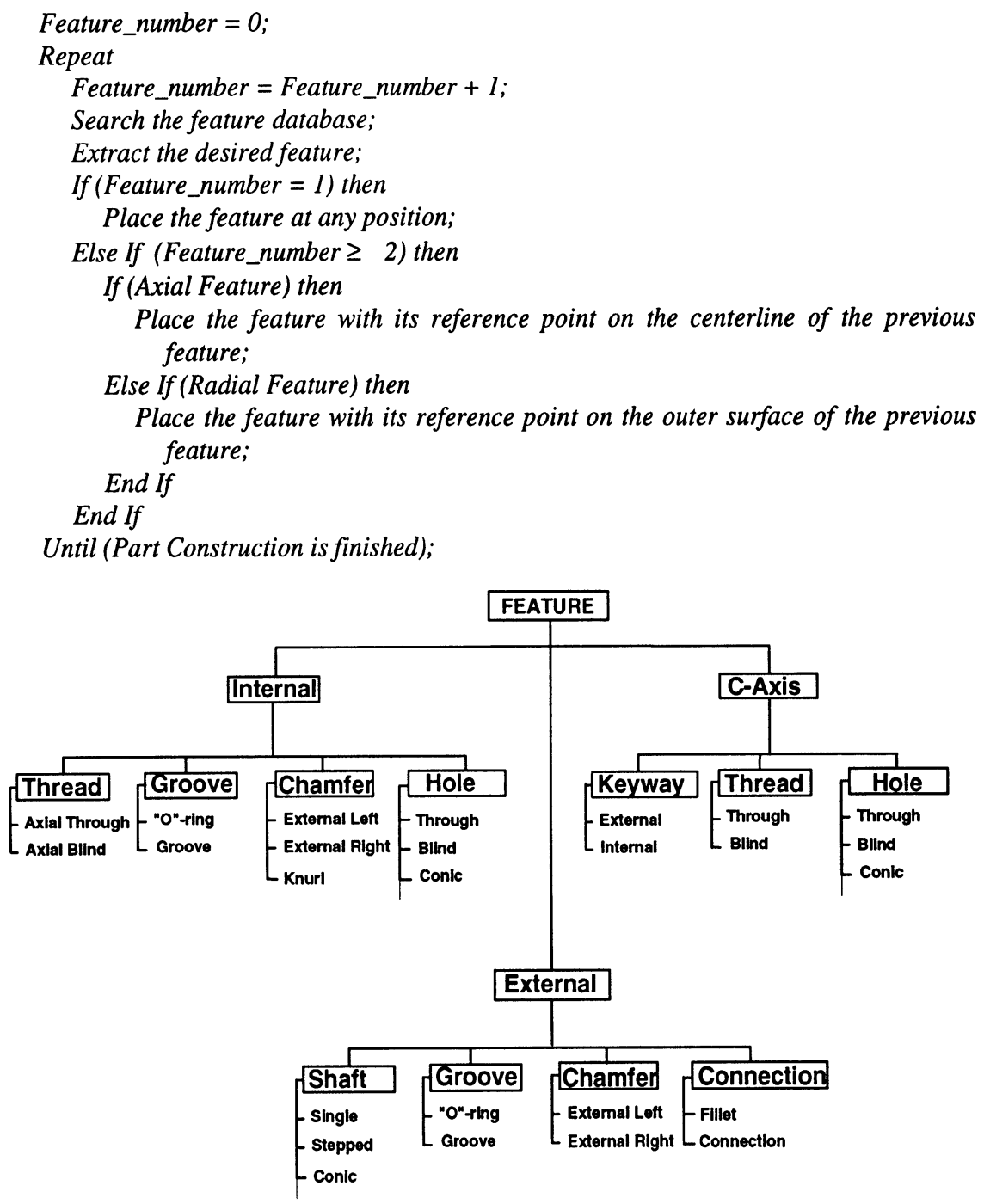

Figure 4 Portion of the feature classification 
Feature type: Symmetrical internal Feature name: Axial eccentric through hole Feature material: SAE 1020 Steel

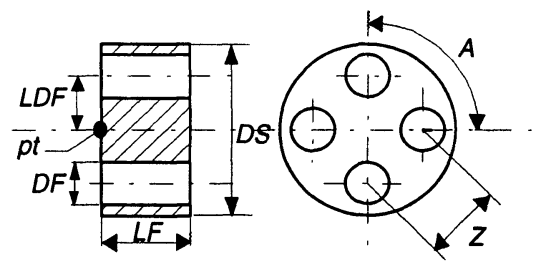

Geometric Parameters:

$L F=$ Hole depth

$L D F=$ Distance between hole center and shaft center $D F=$ Hole diameter

$A=$ Angle between two adjacent holes

$Z=$ Distance between two adjacent holes

$D S=$ Shaft diameter

$p t=$ Reference point

Geometric Constraints:

$0<\mathrm{DF}<(\mathrm{DS} / 2)$

$(\mathrm{DF} / 2)<\mathrm{LDF}<(\mathrm{DS} / 2-\mathrm{DF} / 2)$

$\mathrm{LF}=$ shaft length

$\mathrm{Z}=\mathrm{LDF} \sqrt{2}(1+\cos \mathrm{A}) ; \mathrm{Z}>\mathrm{DF}$

$p t$ should coincide with shaft's reference point

Surface Finish:assigned to internal surface of the hole

Dimensional Tolerances:assigned to DF, LF,LDF

Geometric tolerances: Straightness, Circularity,

Cylindricity, Concentricity, Runout
Feature type: Symmetrical external

Feature name: Shaft with thread

Feature material: SAE 1020 Steel

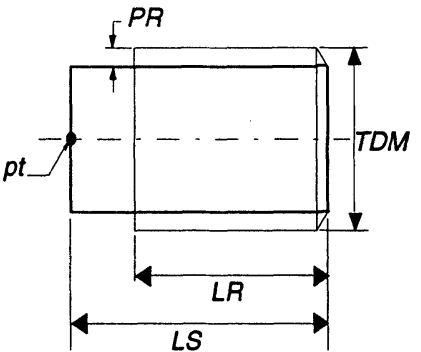

Geometric Parameters:

$L S=$ Shaft length

$L R=$ Thread length

$P R=$ Thread depth

$T D M=$ Thread diameter

$p t=$ Reference point

Geometric Constraints:

Standard DIN 13

$p t$ should coincide with shaft's reference point

Surface Finish: assigned to external surface of the thread

Dimensional Tolerances: assigned to PR, LS, LR and TDM

Geometric Tolerances: Straightness, Circularity, Cylindricity, Concentricity, Runout

Figure 5 Detailed structure of two features present in parts manufactured at the cell

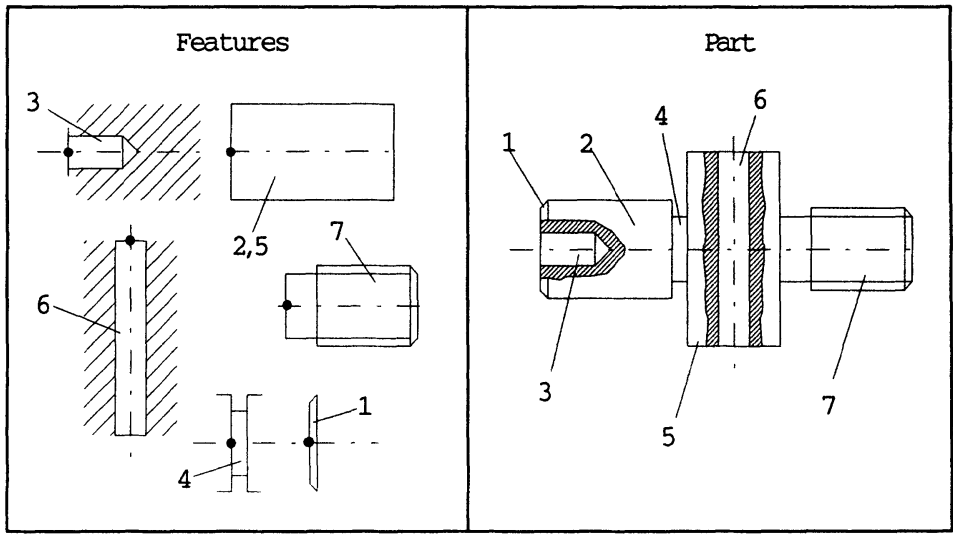

where: 1 = Reference point

Feature $1=$ External chamfer

Feature 2 = Shaft

Feature 3 = Axial concentric blind hole
Feature $4=$ External groove

Feature $5=$ Shaft

Feature $6=$ Radial through hole Feature $7=$ Shaft with thread

Figure 6 Example of part modeling with features 
In Figure 6 an example is given of a part and the features used in its construction.

It should be noticed that the axial features $2,3,4,5$ and 7 have their reference point lying on the centerline of feature 1 . On the other hand, feature 6 , which is a radial feature, has its reference point lying on the outer surface of feature 5 .

Two important characteristics of the CADD module concerning the construction of the part are:

- If the positioning of a feature related to the part violates at least one of the geometric constraints, the CADD module notifies the designer about the inconsistency.

- The designer can choose whether to input the tolerances and surface finish during or after part construction.

The philosophy of the CADD module is similar to the TURBO-MODEL [7], which was also developed taking into account an industrial problem, but CADD gives more flexibility to the designer to construct the part.

FIRST-CUT [13] and the CADD module are different, since in the latter process planning is carried out after the design of the part, which is the approach to design and process planning applied at SLC.

The CADD module of MSSPP is currently being implemented at SLC. This system is being tested by the designers at the company, and it is expected that eventual problems identified by them will be corrected, and suggestions for improvement of the software will be incorporated into the software.

\section{THE EPP MODULE}

The aim of this module is not to automate completely the process planning activity, but rather to provide the process planner with a means for him/her to elaborate consistent and feasible process plans. This module will be responsible for providing the user with the following information: 1-selected blank, 2-selected machine tool, 3-selected cutting tool and workholder, 4-selected machining parameters, 5-time and cost estimates, and 6-process plan.

In order to build the knowledge base in the EPP module, it was necessary to carry out an interview with the process planners of SLC. An example of these rules is given below:

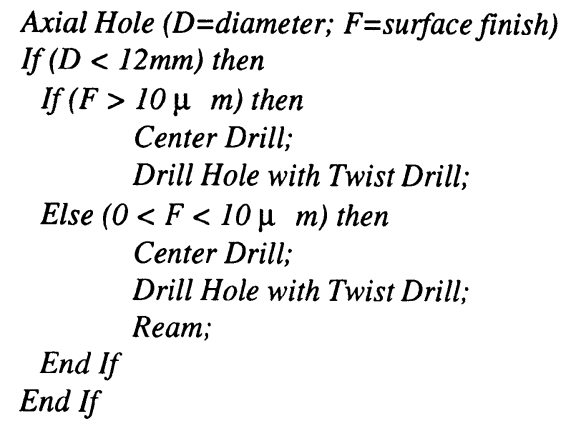

There have been some attempts to standardize process planning information [15,16]. In spite of these efforts, in the development of the EPP module, priority will be given to the information obtained from the SLC process planners, which represent the culture of the company. However, recommendations from outside the company will also be represented in 
the knowledge base, but they will be applied only when the production rules obtained from SLC process planners fail.

The gathering of information necessary for process planning (e.g. machines, tools and fixtures) has been completed, and also the link between this information with the features through their geometrical and technological attributes. However, the EPP module is still to be developed.

\section{THE MANUFACTURING DATABASE}

The information necessary for process plan preparation at SLC is included in the Manufacturing Database (MDB), which is composed of blanks, workholders, machine tools and cutting tools. The structure of the information contained in each of these elements is shown in Figure 7.

The machine utilization parameter is concerned with the percentage of time that the machine tool is being utilized. This information is updated after a certain period of time (e.g. one week, one month, three months, etc.), depending on the company. With this information it is possible to know whether the machine is available at a certain moment or not. This is important for the MSSPP, because the generated process plan should be feasible.

Notice that at the end of its development the manufacturing database will contain the items in the cell considered. In the case of other cells (e.g. cells for prismatic part manufacture), the database will have to be adapted to the blanks, machines, tools and workholders present at the specific cell.

\section{THE CAM MODULE}

This module is concerned with the generation of the tool paths to machine the part. Given the volume to be machined, the machine tool and the cutting tool, this module will generate automatically the speeds, feeds and depths of cut to machine the volume, both for roughing and finishing operations. Also, the tool paths will be simulated on the computer screen. The method to be applied consists of minimizing the cost (or maximize production), having the following as constraints [17,18]: 1-tool life, 2-cutting forces, 3-machine power, 4-cutter deflection, 5-part deflection, 6-surface finish, and 7-workpiece holding stability.

It should be noticed that at SLC, as well as in many other companies, the machining

\begin{tabular}{|l|l|l|l|}
\hline \multicolumn{1}{|c|}{ BLANK } & MACHINE TOOL & WORKHOLDER & CUTTING TOOL \\
\hline Code: & Code: & Code: & Code: \\
Name: & Name: & Name: & Name: \\
Cutter diameter: & Power: & Type: & Cutting Speed: \\
Length: & Table feed: & Max. Clamping Dia.: & Feed-rate: \\
Chemical composition: & RPM: & Workholder diameter: & Depth of cut: \\
Manufacturing method: & No. turret positions: & & Nose radius: \\
Shape: & Bed ways dimensions: & & Diameter: \\
Quantity in storage: & Machine utilization: & & Hardness: \\
& & & Operations: \\
& & & Recommended material: \\
\hline
\end{tabular}

Figure 7 Information on blanks, machines, workholders and tools 
parameters are determined typically from the following sources: tool manufacturer catalogs, machining data handbooks or process planner's experience. However, these parameters are usually conservative for the company's specific equipment. This is why an optimization program will be developed in the CAM module, which will take into account the equipment and the environment at SLC. This will result in more appropriate parameters for the operations, and it is expected that higher production rates and lower costs will be attained.

\section{THE MANUFACTURING SUPPORT SYSTEM MANAGER}

The main attribute of the Manufacturing Support System Manager is to enable a proper communication between each of the modules. This is necessary because the information in each module is represented in different ways, which are usually not compatible. For example, the CADD Module will produce drawing files in a specific CAD format, which may not be standard (e.g. AutoCAD $d w g$ format), and so the CADD module would then have to write the drawing information in a standard format (e.g. IGES). The same applies to the Manufacturing Database, whose information can be created and managed by any commercial database management software. Since the EPP module needs information both from the CADD module and from the MDB module, the MSSM would read the standard part file, which would contain feature information, and also the MDB file. These files would then be translated into an "understandable" format by the MSSM and fed to the EPP module.

If a process plan is successfully generated by the EPP module, the MSSM can then proceed to the generation of the tool path. In the case the EPP module fails in generating a process plan for the given part, the system would notify the user that it failed, and why it happened.

\section{CONCLUSIONS}

The cooperation between UFSC and SLC has been quite encouraging on both sides. On the company's side, the resulting process planning system will assist their process planners to prepare consistent, feasible and reliable process plans in a shorter period of time. Moreover, there will be no need for the process planner to check frequently for the items in storage, machines, tools and workholders available, resulting in a higher job satisfaction and productivity.

On the university side, students become more motivated to work on a project which will result in a product that will be implemented and used in a day to day basis in industry. The students have a practical problem in their hands, and search for realistic solutions.

The feature-based design in the CADD module is one of the most important tasks in the development of this system, because it is the system's "front door". Since this system is to be used in a company, in order for the designers and process planners to became quickly acquainted to it, it should be as friendly as possible. It is thought that with the approach described in this paper this target will be met.

When comparing the systems pointed out above with the CADD module of MSSPP, the latter presents the following advantages:

- It allows a complete definition of the parts present in the application domain, both geometrically and technologically;

- It does not allow a redundant model, as well as a model with geometrical errors;

- Feature-instantiation is easy, which gives flexibility to the designer;

- Since it is connected to a manufacturing database, it enables the designer to look into the raw materials and the manufacturing resources during the design phase; 
- The CADD module can be used in any system that utilizes AutoCAD for part design.

One could argue that the reduction of domain to one cell in the whole factory may hinder the portability of the MSSPP to other cells at SLC. That may be true when considering for instance a cell for manufacturing prismatic parts, where probably in the CADD module the parts would be represented by solid modelling. However, it is thought that, with the knowledge of the company's culture and organization, and after the experience of applying the methodology above to this system, other systems could be developed for other cells, in a shorter time. The system's modularity facilitates the adaptation of each module to the new situation.

In case another company wishes to use the software, it is important that its production is of the batch type, which is the case of most industries today. It is likely that the software would need to be altered in order to incorporate the culture of the new company (e.g. feature information, machine tool and cutting tool information, etc.).

Since the MSSPP is under development, no results have been obtained so far, and therefore effective results of the system, after its implementation at the company, will be reported when that occurs.

\section{EQUIPMENT AND SOFTWARE USED}

The system as a whole is being developed in a 386 microcomputer, under DOS operating system. AutoCAD is being used in the CADD module. IGES is the standard format for interfacing MSSM and CADD. The EPP module will be developed in an expert system shell, based on the " $\mathrm{C}$ " language, which provides the tools necessary for attaining the required efficiency of process planning. The other modules are being developed in the " $\mathrm{C}$ " language.

\section{ACKNOWLEDGEMENTS}

We would like to thank the staff at the Schneider Logemann Cia. (SLC) for their support, without which this work would not have happened. The first author would like to thank "CNPq" (Conselho Nacional de Desenvolvimento Cientifico e Tecnologico) of Brazil, for the scholarship provided.

\section{REFERENCES}

1. Wang, H.P. and Li, J.K. (1991) Computer-Aided Process Planning. Elsevier Science Publishers.

2. Wysk, R.A. (1977) An Automated Process Planning and Selection program: APPAS. Ph.D. Thesis, Purdue University, West Lafayette, Indiana, U.S.A.

3. Van't Erve, A.H. (1988) Generative Computer Aided Process Planning for Part Manufacturing - An Expert System Approach. Ph.D. Thesis, University of Twente, Enschede, The Netherlands, January.

4. Link, C.H. (1976) CAPP - CAM-I Automated Process Planning System, in Proceedings of the 13th Numerical Control Society Annual Meeting and Technical Conference, Cincinatti, Ohio, U.S.A., March.

5. Eversheim, W. , Fuchs, H. and Zons K.H. (1980) Automatic Process Planning with Regard to Production by Application of the System AUTAP for Control Problems, in Computer 
Graphics in Manufacturing Systems, 12th CIRP International Seminar on Manufacturing Systems, Belgrade, Yugoslavia.

6. ElMaraguy, H.A. (1993) Evolution and Future Perspectives of CAPP. Annals of the CIRP, 42, 2, 739-751.

7. Jasthi, S.R.K. et al. (1994) A Feature-Based Part Description System for Computer-Aided Process Planning. Journal of Design and Manufacturing, 4, 67-80.

8. Wang, H.P. and Wysk, R.A. (1988) A Knowledge-Based Approach for Automated Process Planning. International Journal for Production Research, 26, 6, 999-1014.

9. Hinduja, S. and Barrow, G. (1986) TECHTURN: A Technologically-Oriented System for NC Lathes, in 1st Conference on Computer-Aided Production Engineering, Edinburgh, UK, 295-305.

10. Korde, U.P. (1992) Computer-Aided Process Planning For Turned Parts Using Fundamental and Heuristic Principles. Journal of Engineering for Industry, 114, February, 31-40.

11. Shah, J.J. and Rogers, M.T. (1988) Functional Requirements and Conceptual Design of the Feature-Based Modelling System. Computer-Aided Engineering Journal, February, 915.

12. Gu, P. (1994) A Feature Representation Scheme for Supporting Integrated Manufacturing. Computers in Industrial Engineering, 26, 1, 55-71.

13. Cutkosky, M.R. and Tenembaum, J.M. CAD/CAM Integration Through Concurrent Process and Product Design.

14. Juri, A.H. et al. (1990) Reasoning About Machining Operations Using Feature-Based Models. International Journal for Production Research, 28, 1, 153-171.

15. Butterfield, W.R.; Green, M.K.; Scott, D.C. and Stoker, W.J. (1986) Part Features for Process Planning. CAM-I Technical Report R-86-PPP-01, Arlington Texas, U.S.A.

16. ISO TC184/WG3 N324 - T7 (1994) ISO 10303 - Part 224 - Mechanical Product Definition for Process Planning using Form Features, South Carolina, U.S.A., 27/June.

17. Huang, H. (1988) A Generative Process Planning System for Turned Components. Ph.D. Thesis, UMIST, Manchester, UK.

18. Lau, T.L. (1987) Optimization of Milling Conditions. Ph.D. Thesis, UMIST, Manchester, UK. 